\title{
Investigation of interaction of human platelet membrane components with anticoagulant drugs Abciximab and Eptifibatide
}

\author{
Ewa Gorodkiewicz ${ }^{1}$, Anna Sankiewicz ${ }^{1}$, Zbigniew A. Figaszewski ${ }^{1,2}$ \\ ${ }^{1}$ Department of Electrochemistry, Institute of Chemistry, University of Bialystok, Bialystok, Poland \\ ${ }^{2}$ Laboratory of Electrochemical Power Sources, Faculty of Chemistry, University of Warsaw, Warsaw, \\ Poland
}

\begin{abstract}
Abciximab (Abci) and eptifibatide (Epti) are antiaggregate drugs which may reduce thrombotic complications in acute coronary syndromes. The aim of this work was the investigation of the interaction between the phospholipidGPIIb/IIIa glycoprotein complex and Abci or Epti, and the influence of these drugs on the phospholipid ratio in the platelet membrane. The interaction between the phospholipid-GPIIb/IIIa glycoprotein complex and antiaggregate drugs were investigated using the Surface Plasmon Resonance Imaging technique (SPRI). Phospholipids phosphatidylinositol (PI), phosphatidylserine (PS), phosphatidylethanolamine (PE), phosphatidylcholine (PC) and sphingomyelin (SM) were first immobilized onto the gold chip surface. The phospholipid ratio in the platelet membrane was determined by the HPLC. Only PI, PS, PE and PC were determined. Human platelets treated 'in vitro' with Abci or Epti exhibit changes in the phospholipid ratio in the platelet membrane. The ratio of PS decreases and PC rises. The SPRI distinctly shows interactions between phospholipids and glycoprotein GPIIb/IIIa, and between the phospholipid-glycoprotein GPIIb/IIIa complex and Abci or Epti. The interaction between phospholipids and glycoprotein GPIIb/IIIa is growing in the sequence: $\mathrm{PI}<<\mathrm{SM}<\mathrm{PE}<\mathrm{PC}<\mathrm{PS}$. The interaction between phospholipid-glycoprotein GPIIb/IIIa complex and Abci/Epti is growing in the sequence: $\mathrm{PS}<\mathrm{PI}<\mathrm{PC}<\mathrm{PE}<\mathrm{SM}$. SPRI was proved to be excellent tool for observation of such interactions.
\end{abstract}

Key words: abciximab, eptifibatide, phospholipids, SPRI, HPLC

\section{Introduction}

Abciximab and eptifibatide are antiplatelet drugs which may reduce thrombotic complications in acute coronary syndromes or after percutaneous coronary interventions. Primarily, the function of human blood platelets is the maintenance of haemostasis and arresting blood loss from the vessel. The fundamental part of this process is platelet aggregation. However, this aggregation is also the reason for thrombus (blood clot) formation within the coronary artery, especially in patients with acute coronary syndromes.

Blood platelets (thrombocytes) are an important blood component. They play a significance role in the

Correspondence: E. Gorodkiewicz, Dept. of Electrochemistry, Institute of Chemistry, University of Bialystok,

Al. J. Pilsudskiego 11/4, 15-443 Bialystok, Poland;

tel.: (+4885) 7457601, fax.: (+4885) 6647489,

e-mail: ewka@uwb.edu.pl process of thrombosis, hemostasis, clot retraction, host defense, inflammation, tumor growth or metastasis, maintenance and regulation of vascular tone [1]. Human blood platelets (PLT) are small non-nucleated blood cells (approximately 2.0-4.0 $\mu \mathrm{m}$ in diameter). Platelets are generated in the bone marrow by the fragmentation of megakaryocyte [2] and circulate in the organism for 7-10 days [3]. The platelets are composed of platelet organelles such alpha and dense granule, mitochondria, lysosome, as well as an open canalicular system and tubular system, cytoplasm, granules of glycogen and plasma membrane surrounding the glycocalyx $[4,5]$. The glycocalyx contains glycoprotein, glycolipids, mucopolysacharides and adsorbed plasma proteins [6]. Sialic acid is the glycocalyx component. The sialic acid causes a negative charge of the platelet surface [7]. The glycoproteins on the platelet surface are various receptors for adhesive agents, aggregating agents, inhibitors and procoagulant factors. For example, GP Ia/IIa and GP VI are col- 
lagen receptors, GP IIb/IIIa is a fibrinogen receptor, as well as a von Willebrand factor receptor [5].

The platelet plasma membrane is a bilayer of phospholipids with embedded proteins and cholesterol. The phospholipid distribution in the platelet plasma membrane is asymmetric [8]. Generally, in normal nontreated platelets, $90 \%$ of sphingomyelin (SM), $45 \%$ of phosphatidylcholine (PC) are in the outer monolayer, and $90 \%$ of phosphatidylserine (PS), $80 \%$ of phosphatidylethanoloamine and most of the phosphatidylinositol are in the inner monolayer [9].

The platelet aggregation process occurs in two steps: platelet adhesion and platelet activation. After adhesion, the platelet becomes activated involving a series of morphologic and functional changes. The activated platelet secrete the contents of the granule and assumes an irregular shape with many extending pseudopodia $[10,11]$. The platelet plasma membrane with glycoproteins on its surface plays a very important role in this process. The platelet phospholipids are substrates for the formation of arachidonic acid. This acid is the precursor of trombooxane (TXA2). Platelet activation involves changes in membrane phospholipid orientation: the phospahatidylserine (PS) and phosphatidylethanoloamine (PE) surface expression is observed. These changes are the reason for the procoagulant activity platelet surface [12].

Platelet aggregation is mediated by the glycoprotein GPIIb/IIIa receptor. This receptor consists of two subunits, $\alpha \mathrm{II}_{\mathrm{b}}$ and $\beta_{3}$, and require $\mathrm{Ca}^{2+}$ for maintaining the heterodimeric structure of the complex. The alpha subunit is of $136-\mathrm{kDa}$ and consists of a heavy chain and a light chain. The heavy chain is entirely extracellular. The light chain has a short cytoplasmic tail, a transmembrane region, and a small extracellular domain. These two polypeptide chains are linked by a disulfide bond. The beta subunit is of $92 \mathrm{kDa}$ and consists of a single polypeptide chain, with a short cytoplasmic tail, a single transmembrane region, and a large extracellular domain [13]. Platelet activation causes conformational changes in the GP IIb/IIIa receptors, transforming the receptors from a ligand-unreceptive to a ligand-receptive state [14]. This glycoprotein is the most abundant platelet receptor and a final step in thrombous formation, providing the unique possibility of designing agents that specifically block the GPIIb/IIIa receptor and prevent platelets from sticking together and causing thrombus (blood clot) formation within the coronary artery. This, especially in patients with acute coronary syndromes [15]. Currently, there is a class of parenteral drugs that specifically inhibit glycoprotein GPIIb/IIIa. Three of them: the humanized Fab fragment of the monoclonal antibody c7E3 i.e. abciximab (ReoPro), a cyclic heptapeptide eptifibatide (Integrilin) and synthetic nonpeptide analog tirofiban (Aggrasrat) have been approved for clinical use [16-18].
These drugs bind to the glycoprotein GPIIb/IIIa and block the active centre receptor preventing the binding of fibrinogen, the von Willebrand factor, and other adhesive ligands to this receptor.

Abciximab (M=48kDa) [19] is a Fab fragment of a humanized monoclonal antibody c7E3. Abciximab binds to the $\alpha \operatorname{IIb} \beta$ receptor through two binding sites: RGD (Arg-Gly-Asp) and KQAGDV( Lys-Gln-AlaGly-Asp-Val). This is a large molecule and the inhibitory effect of abciximab may be due to the steric hindrance of the ligand access [20]. Abciximab has a short plasma half-life but a long duration of action as a result of its high affinity for the receptor. Platelet function recovers within 24-48 hours after discontinuation of this agent [21].

Eptifibatide is a cyclic heptapeptide containing six amino acids with a characteristic amino acid sequence Lys-Gly-Asp (KGD) and one mercaptopropionyl (desamino cysteinyl) residue. It is a relatively small molecule $(\mathrm{M}<1 \mathrm{kDa})$ [19] that fits into the RGD motif binding pocket of glycoprotein GPIIb/IIIa and blocks the fibrinogen junction to the receptor [22]. Eptifibatide is characterized by a short half-life and normal platelet function is restored within 2 to 4 hours after treatment termination [21].

Platelet functions were investigated in many respects. Blending time, percentage of aggregation platelet, ultrastructural evaluation of platelets $[1,23]$ were investigated, as well as the composition and organization of the platelet membrane lipid $[9,24,25]$, and the interaction between platelet membrane components [26-28] and drugs [29]. The interaction between proteins and lipids was observed by negative staining electron microscopy [26], capillary electrophoresis with laser-induced fluorescence detection [30] and Surface Plasmon Resonance [31,32]. Chromatography methods such as two dimensional thin-layer chromatography [33], high performance thin layer chromatography [34], or high performance liquid chromatography [35] were used for analysis of the phospholipid composition of platelet membranes.

The aim of this work was the investigation of the interaction between the phospholipid-GPIIb/IIIa glycoprotein complex and antiaggregate drugs such as abciximab or eptifibatide, as well as the influence of these drugs on the phospholipid ratio in the platelet membrane.

\section{Materials and methods}

Measuring apparatus. HPLC analysis was done using a Merck Hitachi liquid chromatograph with a diode array detector (model L-4500, Tokyo Japan). A Superspher Si 60 column $(250 \times 4 \mathrm{~mm})$ was applied.

SPRI measurements were performed as described in a previous paper [36-38]. Briefly, the measurements were performed at a fixed angle of incident light and reflectivity was simultaneously measured across an entire chip surface. The contrast values 
obtained for all pixels across a particular sample spot were integrated. Thus the SPRI signal was integrated over the spot area. NIH Image J version 1.32 software was used to evaluate the SPRI images in $2 \mathrm{D}$ form.

Chemicals. Phosphoric acid, sodium chloride, sodium hydrogen carbonate, sodium dihydrogen phosphate, potassium chloride, magnesium chloride, chloroform (all analytical grade, $\mathrm{POCH}$, Gliwice, Poland), Tyrode buffer ( $12 \mathrm{mM} \mathrm{NaHCO}_{3}, 4 \mathrm{mM} \mathrm{NaH} \mathrm{PO}_{4}$, $137 \mathrm{mM} \mathrm{NaCl}, 2,6 \mathrm{mM} \mathrm{KCl}, 1 \mathrm{mM} \mathrm{MgCl}, \mathrm{pH} 7.3$ ), butylated hydroxytoluene (BHT), 1-octadecanethiol ODM (analytical grade SIGMA-ALDRICH), acetonitrile, methanol, n-hexane, isopropanol (all gradient grade for chromatography, MERCK), absolute ethanol (Eurochem BGD, Tarnow, Poland), phospholipids standards- phosphatidylinositol (porcine liver), phosphatidylserine (bovine brain), phosphatidylethanolamine (porcine liver), phosphatidylcholine (porcine liver) and sphingomyelin (porcine liver) ((Larodan AB, Sweden), abciximab (REOPRO, Centocor B.V. Holand), eptifibatide (INTEGRILIN, Glaxo Group Ltd, Great Britain), glycoprotein GPIIb/IIIa (CALBIOCHEM, Merck group, Merck Sp. z.o.o., Warsaw, Poland), photopolymer ELPEMER SD 2054, hydrophobic protective paint SD 2368 UV SG-DG (PETERS, Kempen, Germany) were used as received. Water was deionized with a MiliQ (Milipore) apparatus.

Chip preparation. Gold chips were manufactured as described in the previous paper [36].

Photopolymer deposition. Gold chips were rinsed with ethanol and dried under a stream of nitrogen. A photopolymer layer was then deposited over the gold layer by a screen printing technique. Photopolymer was dried at $70^{\circ} \mathrm{C}$ for 30 minutes and irradiated with UV light $(800 \mathrm{~W})$ from a distance of $30 \mathrm{~cm}$ for 2 minutes. The photopolymer was developed with $1 \%$ aqueous sodium carbonate, washed with redistilled water and dried. The photopolymer layer was covered with a hydrophobic protective paint, as well as by application of the screen printing technique. The paint was irradiated for 5 minutes with a UV lamp $(800 \mathrm{~W})$. Free gold surfaces of the obtained sensor were separated with a photopolymer and hydrophobic paint. Therefore, four different solutions can be simultaneously measured without mixing the tested solutions.

Phospholipids immobilization. Chips with a photopolymer grid were rinsed with ethanol and water and dried under a stream of nitrogen. They were then immersed in 2,7 mM ODM ethanolic solutions for at least $24 \mathrm{~h}$. The chips were further rinsed with ethanol and water and dried under a stream of nitrogen. The phospholipids solutions $(10 \mathrm{mM})$ were prepared in a chloroform-methanol mixture $(2: 1, \mathrm{v} / \mathrm{v})$. These solutions were evaporated to dryness and dissolved in $200 \mu \mathrm{l}$ of water under energy shaking. The phospholipid suspension was placed on the chips for at least $24 \mathrm{~h}$.

Procedure of interaction study. The chips with the phospholipid layer were washed 3 times with water and dried under a stream of nitrogen. The aqueous glycoprotein IIb/IIIa solution $(\mathrm{C}=50 \mu \mathrm{g} / \mathrm{ml})$ was placed on the chips and incubated at $37^{\circ} \mathrm{C}$ for $1 \mathrm{~h}$. The surface of the chips was washed 3 times with water. The chips were placed on the prism in the apparatus and then the SPR signal was measured. It was the signal before the interaction of the phospholipid-glycoprotein complex with a drug. A drop of drug solution (abciximab $\mathrm{C}=3.57 \mu \mathrm{g} / \mathrm{ml}$; eptifibatide $\mathrm{C}=2.56 \mu \mathrm{g} / \mathrm{ml}$ ) was located on the chips for $30 \mathrm{~min}$ of interaction time. The drug solution was removed, the chip surface was washed with the buffer and the SPR measurement was performed again.

Isolation and analysis of platelet phospholipids by HPLC methods. Human platelets were prepared from whole blood, taken from healthy volunteers supplied by the Blood Donor Centre in Bialystok. The blood was incubated with drugs at a therapeutic concentration: abciximab $(3.57 \mu \mathrm{g} / \mathrm{ml}$ of blood) for 0.5 hour,
4 hours, 24 hours or eptifibatide $(2.57 \mu \mathrm{g} / \mathrm{ml}$ of blood $)$ for 0.5 hour, 1 hour and 4 hours. The platelet rich plasma was separated by centrifuging blood at $130 \mathrm{~g}$ for $8 \mathrm{~min}$ at room temperature. This platelet rich plasma was then centrifuged at $2200 \mathrm{~g}$ for $10 \mathrm{~min}$. to obtain a platelet bottom. The supernatant was removed. The platelet bottoms were washed three times with isotonic saline.

The lipid fraction was extracted by using $15 \mathrm{ml}$-hexane-isopropanol mixture $(3: 2, \mathrm{v} / \mathrm{v})$ [39]. The whole, after stirring and shaking, was centrifuged at $4000 \mathrm{~g}$ for $20 \mathrm{~min}$. The lipids extract was evaporated to dryness under nitrogen $\left(\mathrm{N}_{2}\right)$. To prevent the oxidation of phospholipids during extraction, $0.03 \%$ of BHT was added, along with flushing with nitrogen at each step of the procedure.

Individual phospholipids were separated by liquid chromatography in a normal phase system (NP-HPLC) with column Si60, UV detection and isocratic elution. The mobile phase was an acetonitrile-methanol-85\% phosphoric acid mixture of 130:5:1.5 volume ratio. The flow-rate of the mobile phase was $1 \mathrm{ml} / \mathrm{min}$. Chromatogram was recorded at $214 \mathrm{~nm}$ wavelength [40]. The peaks of phosphatidylinositol (PI), phosphatidylserine (PS), phosphatidylethanolamine (PE) and phosphatidylcholine (PC) are well separated.

Statistical analysis. The results are expressed as mean values \pm SD $(n=9$, abciximab; $n=13$, eptifibatide). Statistically significant differences were determined by the Student's t-test, as well as ANOVA. $\mathrm{p} \leq 0.05$ was considered statistically significant.

\section{Results and discussion}

\section{SPR investigation of phospholipids-GP IIb/IIIa-drugs interactions}

The purpose of these experiments was to exhibit interactions between various immobilized phospholipids (PI, PS, PE, PC, SM) and glycoprotein GPIIb/IIIa, as well as the interaction between the immobilized phospholipid-glycoprotein GPIIb/IIIa complex and antiplatelet drugs abciximab or eptifibatide. Phosphatidylinositol (PI), phosphatidylserine (PS), phosphatidylethanolamine (PE), phosphatidylcholine (PC) and sphingomyelin (SM) were first immobilized onto the chip surface. The increase in the signal was observed after interaction of each phospholipid with glycoprotein, showing their mutual interactions. Human albumin was used in the investigation as a comparative protein. When human albumin was used, no binding of any type of phospholipids was observed. The sequence of GPIIb/IIIa affinity to the investigated immobilized phospholipids is: $\mathrm{PS}>\mathrm{PC}>\mathrm{PE}>\mathrm{SM}>>\mathrm{PI}$.

The greatest increase in the SPR signal was observed with phosphatidylserine, which indicates that glycoprotein exhibits the biggest affinity with these phospholipids. Phosphatidylserine is a very important phospholipid in the process of platelet activation and aggregation. The increase in the negative charge of the membrane surface is necessary for the achievement of procoagulant properties. This condition is met when negatively-charged PS is transported from the inner monolayer of cell membrane to its outer monolayer $[12,41,42]$. Other phospholipids are capable of modulating the PS coagulant effect. According to 


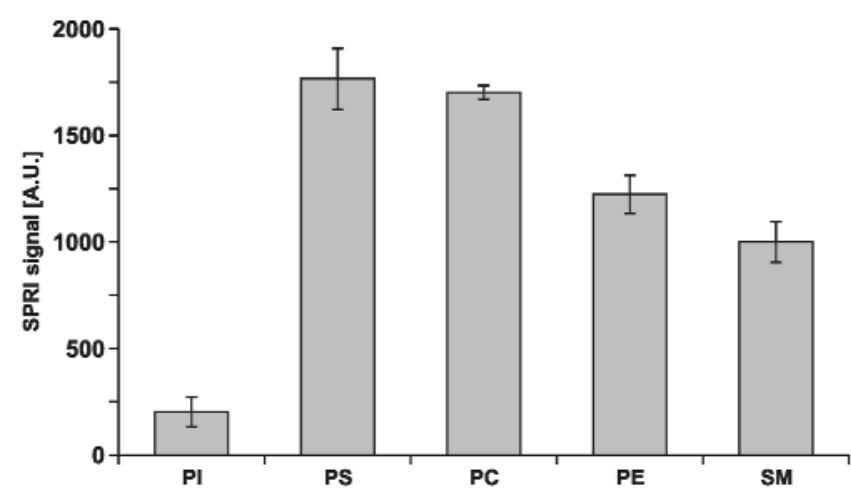

Fig. 1. Increase in the SPR signal after interaction of immobilized phospholipids with glycoprotein GPIIb/IIIa. PI - phosphatidylinositol, PS - phosphatidylserine, PC - phosphatidylcholine, PE phosphatidylethanolamine, and SM - sphingomyelin.

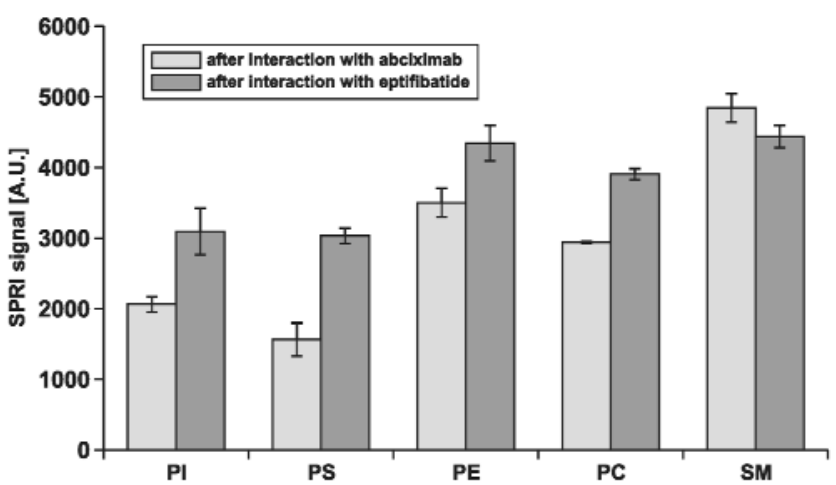

Fig. 2. Increase in the SPR signal after interaction of immobilized phospholipid- complex glycoprotein GPIIb/IIIa with abciximab or eptifibatide. PI - phosphatidylinositol, PS -phosphatidylserine, PC - phosphatidylcholine, PE - phosphatidylethanolamine, and SM sphingomyelin.

Heemskerk [43], PE strengthens the procoagulant properties of the membrane whereas SM weak them. According to Parise and Philips [26] GPIIb/IIIa is the most easily incorporated into the vesicles with the biggest content of PS in the presence of PC. These authors supposed that one of the reasons for strong PS interactions with GPIIb/IIIa may be interactions between an acidic group of PS and the positive charge of GPIIb/IIIa. Negatively charged membrane phospholipids enable dense parallel protein sticking and anchoring in the membrane. PS may cause the defect in the bilayer membrane structure, which causes better GPIIb/IIIa anchoring [26]. Sequence of GPIIb/IIIa affinity to other phospholipids is as follows: $\mathrm{PC}>\mathrm{PE}>\mathrm{SM}$. The percentage of $\mathrm{PC}$ in membrane phospholipids is the greatest (about 40\%) [44].

The data found in the literature $[26,45]$ suggest that interactions PE with certain receptors is similar to PS. $\mathrm{PE}$ is moved to the outer part of membrane during the activation process.

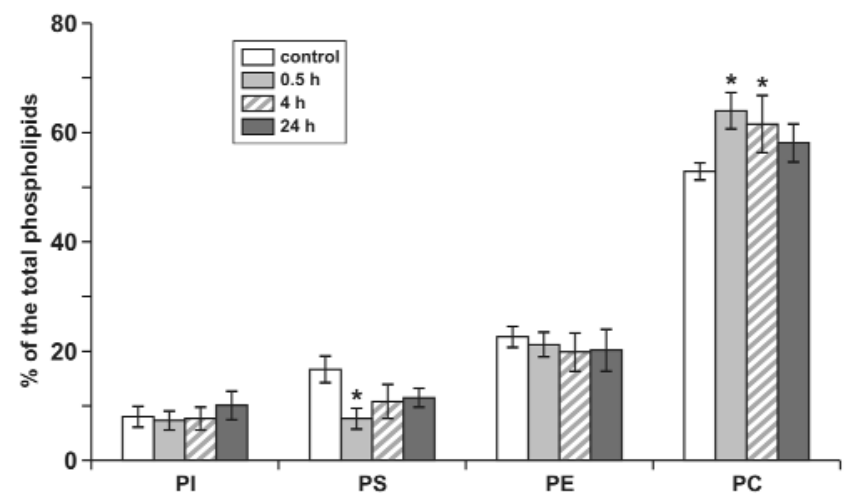

Fig. 3. Comparison of the phospholipid content in platelet membranes as a percentage of the total phospholipids in platelets treated and untreated with abciximab $(\mathrm{n}=9)$. Statistically significant differences for $\mathrm{p} \leq 0.05$ with respect to control.

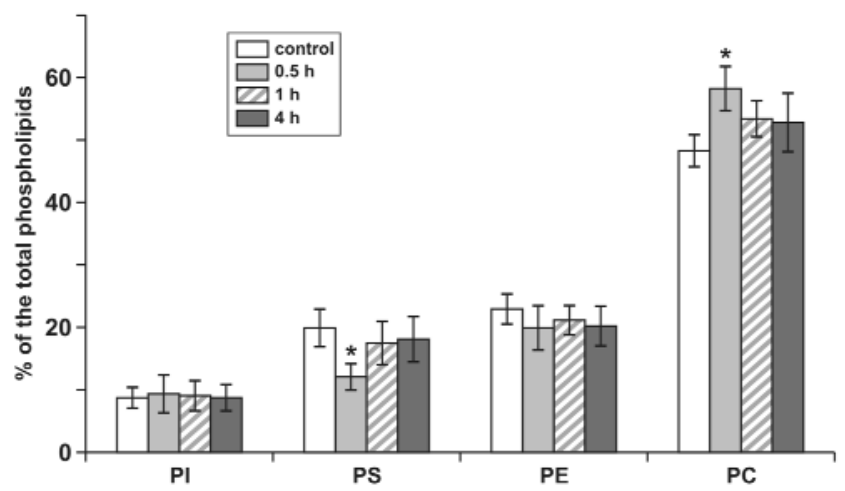

Fig. 4. Comparison of the phospholipid content in platelet membranes as a percentage of the total phospholipids in platelets treated and untreated with eptifibatide $(n=13)$. Statistically significant differences for $\mathrm{p} \leq 0.05$ with respect to control.

The results of the interaction between the immobilized phospholipid-complex glycoprotein GPIIb/IIIa and abciximab or eptifibatide are shown in Figure 2. All complexes exhibit strong interaction with both drugs. This interaction follows the sequence: PS $<$ $\mathrm{PI}<\mathrm{PC}<\mathrm{PE}<\mathrm{SM}$. Strong interactions between both investigated drugs and complexes of phospholipids with glycoprotein GPIIb/IIIa confirm that the role of the drugs is the inactivation of glycoprotein GPIIb/IIIa active areas.

In most cases, the signal increase after interaction of the phospholipid-glycoprotein GPIIb/IIIa complex with the drugs was smaller for abciximab than for eptifibatide.

Eptifibatide is a more specific drug than abciximab. It bonds only with a GPIIb/IIIa receptor [22]. It incorporates into the notch between the arms of GPIIb/IIIa. Several hundred drug molecules join to the single GPIIb/IIIa molecule [46]. Eptifibatide molecules can be combined forming aggregates [11] and can be the rea- 
son for a SPR signal increase dependent on mass. Abciximab incorporates into two active areas, GP RGD and KQAGDU [20]. It is a large molecule and acts as a spherical bloc by covering glycoprotein. 1.5 abciximab molecules joins one GPIIb/IIIa molecule [46].

\section{In vitro investigation of interaction of abciximab and eptifibatide with human platelets in terms of the phospholipid ratio}

Human blood platelets were in vitro treated with anticoagulant drugs abciximab and eptifibatide. After treatment, the phospholipid ratio in cellular membranes was investigated.

Four classes of phospholipids: phosphatidylinositol (PI), phosphatidylserine (PS), phosphatidylethanolamine (PE) and phosphatidylcholine (PC) were isolated from blood platelets and determined using the HPLC method. Under these conditions, sphingomyelin was not determined. The ratio of particular phospholipids in the mixture was calculated as a percentage of the phospholipid peak area in the total area of all peaks.

Figures 3 and 4 show changes in the composition of phospholipids isolated from blood platelets treated with abciximab (Fig. 3) or eptifibatide (Fig. 4).

The investigations demonstrate that both drugs affect the percentage of phosphatidylcholine and phosphatidylserine. There occurs a statistically significant drop of phosphatidylserine level and a concurrent increase in phosphatidylcholine content within a half an hour of the moment of adding the drugs to blood, in comparison to the control sample, which was donor's blood without the drug. In the case of blood incubation with abciximab, PS content decreases by $55 \%$ of the initial value of phospholipids isolated from the sample whereas in the case of eptifibatide decreases by $39.5 \%$. Simultaneously, the increase in the level of PC by approximately $17 \%$ is observed both for abciximab and for eptifibatide. Percentage composition of phosphatidylethanolamine and phosphatidylinositol oscillates within the margin of error. Figs. 3 and 4 indicate that these changes are reversible and the percentage composition of phospholipids gradually reverses. The reversibility of observed changes in the phospholipid ratio is caused by a gradual reduction of the drug activity. Abciximab quickly fixes with the GPIIb/IIIa receptor, concentration of free antibody in blood plasma drops quickly (30 minutes half life). Reversibility of the action is slow and the appropriate function of platelets returns after 24 hours [21]. Eptifibatide fixes to the receptor quickly and disassociates it as quickly, which means that its anti-aggregation effect is diverted after approximately 4 hours [21].

Decreasing PS level and increasing PC level simultaneously indicates that their mutual transformations in a cell are significant. The acceleration of the PS decarboxylation process and its further changes leading to PC formation are likely to occur [47].

Full content of the phospholipid isolate does not change in a statistically significant way.

\section{Conclusions}

Human platelets treated 'in vitro' with anticoagulant drugs abciximab or eptifibatide exhibit changes in the phospholipid ratio in the platelet membrane. The ratio of PS decreases and the ratio of PC rises.

The SPRI technique distinctly shows interactions between phospholipids and glycoprotein GPIIb/IIIa, as well as between the phospholipid-glycoprotein GPIIb/IIIa complex and abciximab or eptifibatide. The interaction between phospholipids and glycoprotein GPIIb/IIIa follows the sequence: $\mathrm{PI}<<\mathrm{SM}<\mathrm{PE}$ $<$ PC $<$ PS. The interaction between the phospholipidglycoprotein GPIIb/IIIa complex and abciximab follows the sequence: $\mathrm{PS}<\mathrm{PI}<\mathrm{PC}<\mathrm{PE}<\mathrm{SM}$, while with the eptifibatide follows the sequence: $\mathrm{PS}, \mathrm{PI}<\mathrm{PC}$ $<\mathrm{PE}<\mathrm{SM}$. Generally, the interactions between the phospholipid-glycoprotein GPIIb/IIIa complex and eptifibatide are stronger than those with abciximab. SPRI was proved to be an excellent tool for the observation of such kinds of interactions.

Acknowledgements: Emilia Kryjan M.Sc. and Ewa Wądołowska M.Sc is acknowledged for his valuable technical assistance.

\section{References:}

[1] Harrison P. Platelet function analysis. Blood Rev. 2005;19(2):111-123.

[2] Patel SR, Hartig JH, Italiano JE. The biogenesis of platelets from megakaryocyte proplatelets. $J$ Clin Invest. 2005;115(12):3348-3354.

[3] Chaer RA, Graham JA, Mureebe L. Platelet Function and Pharmacologic Inhibition. Vasc Endovascular Surg. 2006; 40(4):261-267.

[4] Michelson AD. Platelets. Amsterdam: Elsevier, 2006.

[5] George JN. Platelets. Lancet. 2000;355:1531-1539.

[6] Reitsma S, Slaaf DW, Vink H, van Zandvoort MAMJ, Egbrink MGA. The endothelial glycocalyx: composition, functions, and visualization. Pflugers Arch. 2007;454:345-359.

[7] Kątnik-Prastowska I. Structure and biology of sialic acids. Adv Clin Exp Med. 2003;12(5):653-666.

[8] Chap H, Perret B, Mauco G, Simon MF, Douste-Blazy L. Organization and role of platelet membrane phospholipids as studied with purified phospholipases. Agents Actions. 1979; 9(4):400-406.

[9] Rinder HM, Ault KA. Platelet activation and its detection during the preparation of platelets for transfusion Transfus Med Rev. 1998;12(4):271-287.

[10] Blockmans D, Deckmyn H, Vermylen J. Platelet activation. Blood Rev. 1995;9(3):143-156

[11] Gorodkiewicz E, Sankiewicz A, Sveklo I, Figaszewski ZA. Atomic force microscopy for the characterization of human platelets before and after interaction with selected anticoagulant drugs. Bulg Chem Com. 2009;41:23-30. 
[12] Zwaal RF, Bevers EM, Comfurius P, Rosing J, Tilly RH, Verhallen PF Loss of membrane phospholipid asymmetry during activation of blood platelets and sickled red cells; mechanisms and physiological significance. Mol Cell Biochem. 1989;91(1-2):23-31.

[13] Shah HD, Goyal RK. Glycoprotein IIb/IIIa receptor and its inhibition: A platelet-directed therapeutic strategy. Indian $J$ Pharmacol. 2004;36(3):133-139.

[14] Ma YQ, Oin J, Plow EF. Platelet integrin alpha(IIb)beta(3): activation mechanisms. J Thromb Haemost. 2007;5(7):13451352.

[15] Coller BS. Blockade of platelet GPIIa/IIIb receptors as an antithrombotic strategy. Circulation. 1995;92:2373-2380.

[16] Stevens M. Glycoprotein IIb/IIIa inhibitors. Intens Crit Care Nurs. 2002;18(1):64-66.

[17] Bennet JS. Novel platelet inhibitors. Аnпu Rev Med. 2001;52:161-184.

[18] Topol EJ, Byzova TV, Plow EF. Platelet GPIIb-IIIa blockers. Lancet. 1999;353:227-231.

[19] Dyke CM. Safety of glycoprotein IIb-IIIa inhibitors: A heart surgeon's perspective. Am Heart J. 1999;138(4):307-316.

[20] Kawecka-Jaszcz K, Olszanecka A, Loster M. Działanie niepożądane leków przeciwpłytkowych w kontekście prewencji chorób układu sercowo-naczyniowego. Choroby Serca i Naczyń. 2006;3(2):91-104.

[21] Jay RM, Lui P. How anticoagulants work Techniques in Regional Anaesthesia and Pain Management. 2006;10:30-39.

[22] Phillips RD, Scarborough MR. Clinical pharmacology of eptifibatide. Am J Cardiol. 1997;80(4A):11B-20B.

[23] Cox D. Methods for monitoring platelet function. Am Heart J. 1998;135(5):5160- 5169

[24] Bevers EM, Comfurius P, Zwaal RF Changes in membrane phospholipid distribution during platelet activation: Biochim Biophys Acta.1983;736:57-66.

[25] Bodin S, Tronchere H, Payrastre B. Lipid rafts are critical membrane domains in blood platelet activation processes. Biochim Biophys Acta. 2003;1610:247-257.

[26] Parise LV, Philips DR. Platelet membrane glycoprotein IIb-IIIa complex incorporated into phospholipid vesicles. Preparation and morphology. J Biol Chem. 1985;260(3):1750-1756.

[27] Zwaal RF, Comfurius P, Bevers EM. Lipid - protein interaction in blood coagulation. Biochim Biophys Acta. 1998;1376(3):433-453.

[28] Razmara M, Hu H, Masquelier M, Li N. Glycoprotein IIb/IIIa blockade inhibits platelet aminophospholipid exposure by potentiating translocase and attenuating scramblase activity. Cell Mol Life Sci. 2007;64:999-1008.

[29] Ciborowski M, Tomasiak M. The in vitro effect of eptifibatide, a glycoprotein IIb/IIIa antagonist, on various responses of porcine blood platelets. Acta Pol Pharm. 2009;66(3):235-242.

[30] Hu S, Zhang L, Dovichi NJ. Characterization of the interaction between phospholipid and protein by capillary electrophoresis with laser-induced fluorescence detection. $J$ Chromatogr A. 2001;924(1-2):369-375.

[31] Ying-Jie Lu, Xiao-Feng Xia, Sen-fang Sui. The interaction of trichosanthin with supported phospholipid membranes stud- ied by surface plasmon resonance. Biochim Biophys Acta. 2001;1512:308-316.

[32] Kuziemko GM, Stroh M, Stevens RC Cholera toxin binding affinity and specificity for gangliosides determined by surface plasmon resonance. Biochemistry.1996;35:6375-6384.

[33] Caimi G, Lo Presti R, Montana M, Canino B, Serra A, Catania A. Platelet membrane fluidity and platelet membrane lipid pattern in several clinical conditions. Clin Hemorheol. 1996;16:177-185.

[34] Biró E, Akkerman JWN, Hoek FJ, Gorter G, Pronk LM, Sturk A, Nieuwland R. The phospholipid composition and cholesterol content of platelet-derived microparticles: a comparison with platelet membrane fractions. J Thromb Haemost. 2005;3(12):2754 -2763.

[35] Kawasaki T, Kambayashi J, Mori T, Kosaki G. Analysis of platelet PL by HPLC. Thromb Res.1984;36:335-344.

[36] Gorodkiewicz E. The Surface Plasmon Resonance Imaging Sensor for Papain Based on Immobilized Cystatin. Protein Pept Lett. 2007;14:443-445.

[37] Gorodkiewicz E. Surface Plasmon Resonance Imaging sensor for cathepsin determination based on immobilized cystatin. Protein Pept Lett. 2009;16:1379-1385.

[38] Gorodkiewicz E, Fernández-González A, Akkoyun A, Salzer R. Systematic Evaluation of a Surface Plasmon Resonance Imaging Biochip Reader: Study of Gold Surface Modifications. Chem Anal (Warsaw). 2005;50:103-116.

[39] Radin NS. Extraction of tissue lipids with a solvent of low toxicity. Methods Enzymol. 1981;72:5-7.

[40] Ostrowska J, Skrzydlewska E, Figaszewski Z. Isolation and analysis phospholipids, Chem Anal (Warsaw). 2000; 45:613630.

[41] Zwaal RF. Membrane and lipid involvement in blood coagulation. Biochim Biophys Acta. 1978;515:163-205.

[42] Zwaal RF, Comfurius P, Bevers EM. Mechanism and function of changes in membrane-phospholipid asymmetry in platelets and erythrocytes. Biochem Soc Trans. 1993;21(2):248-253.

[43] Heemskerk JWM, Bevers EM, Lindhout T. Platelet Activation and Blood Coagulation. Thromb Haemost. 2002;88:186193.

[44] Soares J. Reproducibility of in vivo measures of platelet membrane phospholipids in human subjects. Psychiatry Res. 1999;86(2):107-112.

[45] Conforti G, Zanetti A, Pasquali-Ronchetti I, Quaglino DJr, Neyroz P, Dejana E. Modulation of vitronectin receptor binding by membrane lipid composition. $J$ Biol Chem. 1990;265(7):4011-4019.

[46] Tcheng JE. Clinical challenges of platelet glycoprotein IIb/IIIa receptor inhibitor therapy: bleeding, reversal, thrombocytopenia, and retreatment. Am Heart J. 2000;139:538545.

[47] James GA Jr, Kearns MA, Bankaitis VA. Phospholipid metabolism and membrane dynamics. Curr Opin Cell Biol. 1996;8(4):534-541. 